\title{
Does the use of a specialised paediatric retrieval service result in the loss of vital stabilisation skills among referring hospital staff?
}

\author{
P Ramnarayan, J Britto, A Tanna, D Thomas, S Alexander, P Habibi
}

See end of article for authors' affiliations ......................

Correspondence to: Dr J Britto, Department of Paediatrics, Imperial College School of Medicine, St Mary's Hospital, Norfolk Place, London W2 IPG, UK;

j.britto@ic.ac.uk

Accepted

10 February 2003
Aims: To compare the proportion of airway and vascular access procedures performed by referring hospital staff on critically ill children in two discrete time periods, before and after widespread use of a specialised paediatric retrieval service.

Methods: Transport data were obtained from retrieval logs of all children for whom a paediatric retrieval team was launched in each of two time periods (October 1993 to September 1994; and October 2000 to September 2001).

Results: The overall intubation rate was similar in the first and second time periods $(83.9 \% \vee 79.1 \%)$. However, $31 / 51(61 \%)$ retrieved children were intubated by referring hospital staff in 1993-94, compared to $227 / 269(84 \%)$ in 2000-01. Referring hospital staff gained central venous access in $11 \% \mathrm{v}$ $18 \%$ and arterial access in $22 \% \vee 19 \%$ of retrieved children in the first and second time periods respectively. This was in spite of a significant reduction in the proportion of children on whom these procedures were performed.

Conclusion: Referring hospital staff are performing a greater proportion of initial airway and vascular access procedures undertaken in the stabilisation of sick children retrieved by a specialised paediatric retrieval team. The provision of this service has not resulted in the loss of vital skills at the local hospital.
C entralisation of specialist paediatric services has been a continuously evolving process within the National Health Service (NHS) in the past few years. ${ }^{12}$ It has now become apparent that it forms is an inevitable part of the new structure of health care delivery for children. Specifically, centralisation of care within paediatric intensive care (PIC) has been prompted by a combination of accumulating evidence and expert opinion that suggests better patient care as well as improved morbidity and mortality with this model. ${ }^{3-5}$ A British Paediatric Association report in $1993^{6}$ and a Department of Health document from $1997^{7}$ detail the reasons and the template for future development of centralised PIC in the UK. A recent consensus report from the American Academy of Pediatrics and American Society for Critical Care Medicine deals with the logistic issues involved with the process of centralisation and explains the rationale behind these changes in the USA. ${ }^{8}$

Regionalisation of inter-hospital transport of sick children follows as a logical consequence of this process of centralisation. In order to benefit from the advantages of centralised PIC, critically ill children need to be managed in a tertiary centre. However, the majority of critically ill children present acutely to their local district general hospital (DGH) emergency department. A small but significant proportion of these children will require subsequent care on a paediatric intensive care unit (PICU) and are likely to need inter-hospital transfer. In order to maximise patient care, the transport process must provide a standard of care equal to that offered in the PICU at the point of retrieval (the DGH). ${ }^{9}$ There is considerable evidence that this standard can be attained with the use of specialist teams. ${ }^{10}$ There is also evidence that teams unfamiliar with transporting critically ill children do not achieve this standard. ${ }^{11}$ For this reason, the use of specialist paediatric retrieval teams for inter-hospital transport has been increasingly accepted as routine practice, closely mirroring changes in the process of centralisation.
Concerns have been expressed in the past that centralisation of PIC and the use of a specialised retrieval service for inter-hospital transport would lead to unfamiliarity with vital resuscitation and stabilisation skills among staff managing sick children at DGHs. ${ }^{12}$ This would result in the loss of ability to perform these essential procedures. It is possible that with the widespread use of a specialised retrieval service, doctors at the referring hospital may actually wait for the retrieval team to arrive to carry out vital stabilisation procedures such as endotracheal intubation and vascular access; these procedures ought to have been performed as part of the ongoing resuscitation and initial stabilisation of the child. The majority of transfers in the UK occur by road, and median response times for a specialist team to arrive at the patient's bedside may vary from 100 to 105 minutes. ${ }^{101314}$ Deferring stabilisation procedures until the arrival of the retrieval team could adversely impact on patient morbidity.

Our experience suggested that staff in local hospitals have become more proficient in the initial management of sick children, despite the increasing use of a specialised transport team. To clarify these points, we decided to undertake a study involving all children transported to our PICU over two time periods, one before, and one after the widespread implementation of our specialised retrieval service.

\section{METHODS}

Data regarding all aspects of transport have been collected prospectively on our PICU from 1993. This dataset was accessed to provide data relevant to this study, which included details regarding the initial airway and vascular access

Abbreviations: DGH, district general hospital; PIC, paediatric intensive care; PICU, paediatric intensive care unit 
Table 1 Comparison of diagnostic groups in 1993-94 versus 2000-01

\begin{tabular}{lcl}
\hline Diagnosis & $\begin{array}{l}1993-94 \\
(\mathrm{n}=64)\end{array}$ & $\begin{array}{l}2000-01 \\
(\mathrm{n}=353)\end{array}$ \\
\hline Bronchiolitis & $13(20.3 \%)$ & $41(11.6 \%)$ \\
Central nervous system disease & $8(12.5 \%)$ & $69(19.5 \%)$ \\
ENT/respiratory disease (other) & $8(12.5 \%)$ & $99(28 \%)$ \\
Meningococcal disease & $27(42.2 \%)$ & $70(19.8 \%)$ \\
Sepsis (other) & 4 & 27 \\
Cardiac & 1 & 27 \\
Metabolic disease & 0 & 5 \\
Poisoning & 1 & 4 \\
Trauma & 1 & 2 \\
Other & 1 & 9 \\
\hline
\end{tabular}

management procedures undertaken in all children for whom a paediatric retrieval team was launched in two separate one year time periods. The first one year period was October 1993 to September 1994; the second was October 2000 to September 2001 (some of the data included in this study were published in 1995). ${ }^{10}$ These periods were chosen deliberately separated by many years to allow sufficient changes to have occurred within the model of paediatric retrieval services in the Southeast of England. It was postulated that in 1993 (the year in which the British Paediatric Association report was published), a specialist transport team was not widely used by DGHs; whereas in the latter period, most paediatric retrievals were undertaken by such a specialist team.

We used as outcome measures: the proportion of children in whom the DGH staff performed the initial airway management procedure (endotracheal intubation) and vascular access procedures (arterial and central venous access) in each of the two time periods.

Comparison of proportions was performed by using the $\chi^{2}$ test for independence (or Fisher's exact test when one of the sample sizes was small). Results were expressed in terms of odds ratios. Statistical significance was defined as $\mathrm{p}<0.05$.

\section{RESULTS}

Complete data were available on 340/353 children retrieved in 2000-01, compared to 64/65 children retrieved in 1993-94. Reasons for referral covered a wide range of diagnoses. Table 1 provides a breakdown of the diagnostic groups, and shows that respiratory illness and sepsis were the main diagnostic categories $(32.8 \%$ and $48.4 \%$ respectively in $1993-94 ; 39.6 \%$ and $27.5 \%$ respectively in $2000-01$ ).

Table 2 provides a comparison of the two groups of children included in the analysis, with regard to the overall rate of each intervention. The proportion of children retrieved in whom endotracheal intubation was performed did not change significantly in the two time periods $(83.9 \%$ and $79.1 \%$ respectively, $p=0.69$ ). This was in contrast to central venous and arterial access procedures. The proportion of children retrieved in whom a central venous access procedure was performed decreased from $70.3 \%$ in $1993-94$ to $51.8 \%$ in $2000-01$
Table 3 Proportion of endotracheal intubation performed by referring hospital staff among children retrieved

\begin{tabular}{lll}
\hline & $1993-94$ & $2000-01$ \\
\hline Referring hospital staff (\%) & $31(59.6 \%)$ & $227(84.4 \%)^{*}$ \\
Retrieval team & 21 & 42 \\
Total & 52 & 269 \\
\hline * $p<0.001$, OR 3.66, 95\% Cl 1.92 to 6.97. \\
\hline
\end{tabular}

Table 4 Comparison of proportion of central venous access procedures performed by referring hospital staff

\begin{tabular}{lcc}
\hline & $1993-94$ & $2000-01$ \\
\hline Referring hospital staff (\%) & $5(11.1 \%)$ & $33(18.9 \%)^{*}$ \\
Retrieval team & 40 & 142 \\
Total & 45 & 175 \\
\hline
\end{tabular}

${ }^{*} \mathrm{p}=0.31, \mathrm{OR} 1.86,95 \% \mathrm{Cl} 0.68$ to 5.07 .

Table 5 Comparison of proportion of arterial access procedures performed by referring hospital staff

\begin{tabular}{llc}
\hline & $1993-94$ & $2000-01$ \\
\hline Referring hospital staff (\%) & $11(21.1 \%)$ & $45(19.2 \%)^{*}$ \\
Retrieval team & 41 & 189 \\
Total & 52 & 234
\end{tabular}

${ }^{*} p=0.89, \mathrm{OR} 0.89,95 \% \mathrm{Cl} 0.42$ to 1.86

( $p=0.006)$; for arterial access, these proportions were $83.9 \%$ and $69.8 \%$ respectively $(\mathrm{p}=0.024)$.

Table 3 shows the proportion of endotracheal intubation performed by DGH staff in the two time periods. This proportion increased from $59.6 \%$ in $1993-94$ to $84.4 \%$ in 2000-01 ( $p<0.001$, odds ratio 3.66 , 95\% CI 1.92 to 6.97 ). From table 4, it is clear that the proportion of central venous access procedures performed by DGH staff also increased from $11.1 \%$ to $18.9 \%$. However, this change was not statistically significant ( $\mathrm{p}=$ value 0.31 , odds ratio $1.86,95 \%$ CI 0.68 to 5.07 ). Table 5 suggests hardly any change in the proportion of arterial access procedures performed by DGH staff.

\section{DISCUSSION}

The results of this study indicate that the number of retrievals to our unit increased fivefold from 1993-94 to 2000-01; however, a smaller proportion of children retrieved had interventions performed on them. Of these children in the 2000-01 cohort, a greater proportion were intubated and managed with invasive haemodynamic monitoring by DGH staff in the initial few hours preceding arrival of the specialist retrieval team. This confirms that staff at referring hospitals are not

$\begin{aligned} & \text { Table } 2 \text { Comparison of overall rates of intervention in patient groups in the two } \\
& \text { time periods }\end{aligned}$
\begin{tabular}{llll}
\hline & $\begin{array}{l}1993-94 \\
(\text { Intervention/total) }\end{array}$ & $\begin{array}{l}2000-01 \\
\text { (Intervention/total) }\end{array}$ & p value \\
\hline Endotracheal intubation & $52 / 64(83.9 \%)$ & $269 / 340(79.1 \%)$ & 0.69 \\
Central venous access & $45 / 64(70.3 \%)$ & $175 / 338(51.77 \%)$ & 0.006 \\
Arterial access & $52 / 62(83.9 \%)$ & $234 / 335(69.8 \%)$ & 0.024 \\
\hline
\end{tabular}


performing fewer resuscitation and stabilisation procedures during the management of critically ill children than in the past. This trend is most obvious with airway management (endotracheal intubation rate), and less marked for circulatory management (central venous and arterial access). The overall decreased rate of intervention for these latter procedures over the years may be explained by a relative increase in the proportion of children who were intubated, but did not need central venous or arterial access.

Although an index of disease severity (such as PRISM III ${ }^{15}$ or PIM ${ }^{16}$ ) was not used to formally compare the two groups, the rate of endotracheally intubated patients in the cohorts remained similar, suggesting that there was no large change in severity of illness. The assumption that the rate of endotracheal intubation can be used as a surrogate marker for severity of illness has previously been made in a study assessing changes in the profile of PIC associated with regionalisation. ${ }^{17}$

Early stabilisation is a crucial step in the initial management of a sick child presenting to an emergency department. ${ }^{18}$ Failure to stabilise the child may result in continuation of physiological deterioration and an inability to reverse the pathological processes that lead to further patient morbidity and mortality. ${ }^{19}$ We chose airway and vascular access procedures as markers of stabilisation skills in critically ill children. Endotracheal intubation is a vital skill that is commonly employed in airway management in an emergency setting. The ability to achieve central venous access is considered a necessary stabilisation skill: it allows the measurement of central venous pressure to guide further fluid resuscitation and the delivery of multiple infusions as well as inotropic support centrally (such as adrenaline in children with septicaemia). ${ }^{20}$ The ability to perform invasive blood pressure monitoring and measure blood gases regularly makes arterial access an important procedure in the management of a sick child. Therefore, familiarity with these procedures is crucial to the emergency stabilisation of a sick child.

A previous study has shown that physicians at local hospitals continue to perform adequate clinical assessment of critically ill children, ${ }^{21}$ and refer to tertiary centres appropriately. We believe that there are several reasons why DGH staff have retained their assessment and stabilisation skills. An increasing number of DGH staff are now trained in Advanced Paediatric Life Support. Better training of middle grade staff, increasing "hands on" consultant involvement in the emergency department, better organisation of training in paediatric anaesthesia, ${ }^{22}$ and rotation of trained senior specialist registrars to DGHs (previously confined to teaching hospitals) may all play a significant part in accounting for these findings. Active support from the tertiary centre, beginning with telephone advice while the retrieval team is en route, and dissemination of best practice management guidelines to local hospitals, may also play a major part in explaining some of these results. ${ }^{23}$ The role of DGH staff in the management of sick children prior to intensive care needs to be recognised and supported by tertiary PICUs within the model of centralised paediatric intensive care. Training of front line staff at these centres will need to focus on the initial resuscitation and stabilisation of critically ill children.

\section{Limitations of the study}

The increasing use of resuscitation and stabilisation procedures may not, by themselves, adequately represent proficiency in the management of critically ill children by DGH staff. Management of the sick child also includes other vital clinical skills such as early recognition of illness, adequate seizure control, and the institution of appropriate antibiotic therapy. As such, the use of procedural skills as the only markers of management of the child at the referring hospital is difficult to generalise to the overall quality of initial stabilisation. Moreover, the quality of interventions performed at the referring hospital was not examined in this study. It is possible that the increased number of interventions by DGH staff was associated with a higher complication rate. It is also possible that in the absence of the centralised PIC model, quality of initial management at the DGH may have improved even further due to increasing recognition of the need for early and aggressive stabilisation in this subset of children. A study undertaken from the DGH perspective, which examines the number and quality of initial stabilisation procedures in sick children, may answer many of these questions.

This study does not describe the specific designation and specialty breakdown of staff involved in performing the greater proportion of vital procedures. It is highly likely that this increase could all be attributed to the involvement of anaesthetic staff rather than paediatricians; this information would have been useful to examine issues regarding further medical training and education of medical and nursing staff at the DGH.

\section{Conclusions}

The widespread use of a specialist paediatric retrieval service has not resulted in loss of vital stabilisation skills among referring hospital staff. In fact, a greater proportion of initial stabilisation, notably endotracheal intubation, is being performed by staff at the referring hospital. This finding will help to define the future role of DGHs within the evolving model of centralised paediatric care, and provide guidance on issues pertaining to training of front line staff involved in the initial management of the majority of sick children.

\section{Authors' affiliations}

P Ramnarayan, J Britto, A Tanna, D Thomas, S Alexander,

P Habibi, Department of Paediatrics, Imperial College School of Medicine, St Mary's Hospital, Norfolk Place, London, UK

Contributorship: PR and DT conceived the study idea; PR designed the study, analysed the data, and wrote the first and second drafts of the paper; AT collected the data and helped in data analysis; JB collected data and supervised the first draft; $\mathrm{SA}$ and $\mathrm{PH}$ collected the data, helped in data analysis, and supervised the second draft. $\mathrm{PH}$ will serve as guarantor to the paper.

\section{REFERENCES}

1 Simpson AN, Wardrope J, Burke D. The Sheffield experiment: the effects of centralising accident and emergency services in a large urban setting. Emerg Med J 2001;18:193-7.

2 Arul GS, Spicer RD. Where should paediatric surgery be performed? Arch Dis Child 1998;79:65-70.

3 Pollack MM, Alexander SR, Clarke N, et al. Improved outcomes from tertiary centre pediatric intensive care. A statewide comparison of tertiary and nontertiary care facilities. Crit Care Med 1991;19:150-9.

4 Edge WE, Kanter RK, Weigle CGM, et al. Reduction of morbidity in interhospital transport by specialised pediatric staff. Crit Care Med 1994;22:1186-91

5 Pearson G, Shann F, Barry P, et al. Should paediatric intensive care be centralised? Trent versus Victoria. Lancet 1997;349:1213-17.

6 British Paediatric Association. The care of critically ill children. Report of a multidisciplinary working party on intensive care. London: BPA, 1993.

7 National Coordinating Group on Paediatric Intensive Care. Paediatric intensive care: a framework for the future. London: Department of Health, 1997.

8 Society of Critical Care Medicine. Consensus report for regionalisation of services for critically ill or injured children. Crit Care Med 2000;28:236-9.

9 Rashid A, Bhuta T, Berry A. A regionalised transport service, the way ahead? Arch Dis Child 1999:80:488-92.

10 Britto J, Nadel S, Maconochie I, et al. Morbidity and severity of illness during interhospital transfer: impact of a specialised paediatric retrieval team. BM 1995;311:836-9.

11 Barry PW, Ralston C. Adverse events occurring during inter-hospital transfer of the critically ill. Arch Dis Child 1994;71:8-11.

12 Raffles A. Intensive care provided by local hospitals should be improved. BM 1996;312:120.

13 Cray SH, Heard CM. Transport for paediatric intensive care. Measuring the performance of a specialist retrieval service. Paediatr Anaesth 1995:5:287-92.

14 Robb HM, Hallworth D, Skeoch CH, et al. An audit of a paediatric intensive care transfer unit. British Journal of Intensive Care 1992:2:371-9.

15 Pollack MM, Patel KM, Ruttimann UE. PRISM III. An updated pediatric risk of mortality score. Crit Care Med 1996;24:742-52. 
16 Shann F, Pearson G, Slater A, et al. Paediatric index of mortality (PIM): a mortality prediction model for children in intensive care. Intensive Care Med 1997;23:201-7

17 Pearson G, Barry P, Timmins C, et al. Changes in the profile of paediatric intensive care associated with centralisation. Intensive Care Med 2001;27:1670-3.

18 Kronick JB, Kissoon N, Frewen TC. Guidelines for stabilizing the condition of the critically ill child before transfer to a tertiary care facility. Can Med Assoc J 1998;139:213-20.

19 Mayer TA ed. Transportation of the injured child. In: Emergency management of pediatric trauma. Toronto: Saunders, 1985:508-23.
20 Britto J, Habibi P. Stabilisation and transport of critically ill children. In: Recent advances in paediatrics. London: Churchill Livingstone, 1998:85-113

21 Goh AY, Mok Q. Centralisation of paediatric intensive care: are critically ill children appropriately referred to a regional centre? Intensive Care Med 2001;27:730-5

22 Department of Health. A guide to specialist registrar training. London: HMSO, 1996.

23 Booy R, Habibi P, Nadel S, et al. Reduction in case fatality rate from meningococcal disease associated with improved healthcare delivery. Arch Dis Child 2001;85:386-90.

\section{IMAGES IN PAEDIATRICS}

\section{Radiographic long bone appearance in a child administered cyclical pamidronate}

T

he appearance of this radiograph caused considerable concern in the casualty department. It shows the left wrist of an 11 year old girl and was taken follow-

ing suspicion of a fracture. She had been treated for two years with cyclical pamidronate for type 1 osteogenesis imperfecta (OI). There are multiple linear areas of increased bone density at the metaphysis of the distal radius and ulnar, thickened cortices, with evidence of background osteopenia and no fracture. It is likely that the areas of increased bone density correspond to the timings of the pamidronate infusions.

The use of pamidronate for childhood OI has increased because of its proven benefits of increased bone density, reduced fracture rates, and improved bone pain. ${ }^{12}$ For the first time the effects of pamidronate on bone tissue in growing children have been elucidated from bone histomorphometric studies, thus aiding the explanation for the radiographic appearance. ${ }^{3}$ Treatment leads to a marked reduction in bone remodelling activity of trabecular bone which is predominantly found in the metaphysis of long bones. The lines of increased bone density are likely to be the result of an increase in both the number of trabeculae and the amount of residual calcified cartilage within the secondary spongiosa. The thickened cortices result from preserved modelling directed bone formation coupled with inhibition of bone resorption. ${ }^{3}$

Children with OI are likely to present to the casualty department. This radiograph shows the appearance that might be expected in such individuals treated with pamidronate, and does not require further investigation.

J H Davies, J W Gregory

Department of Child Health, University of Wales College of Medicine, Heath Park, Cardiff CF14 4XN, UK daviesih@cf.ac.uk

\section{References}

1 Glorieux FH, Bishop NJ, Plotkin $\mathrm{H}$, et al. Cyclic administration of pamidronate in children with severe osteogenesis imperfecta. N Engl J Med 1998;339:947-52.

2 Astrom E, Soderhall S. Beneficial effect of long term intravenous bisphosphonate treatment of osteogenesis imperfecta. Arch Dis Child 2002;86:356-64.

3 Rauch F, Travers $\mathrm{R}$, Plotkin $\mathrm{H}$, et al. The effects of intravenous pamidronate on the bone tissue of children and adolescents with osteogenesis imperfecta. J Clin Invest 2002;110:1293-9.

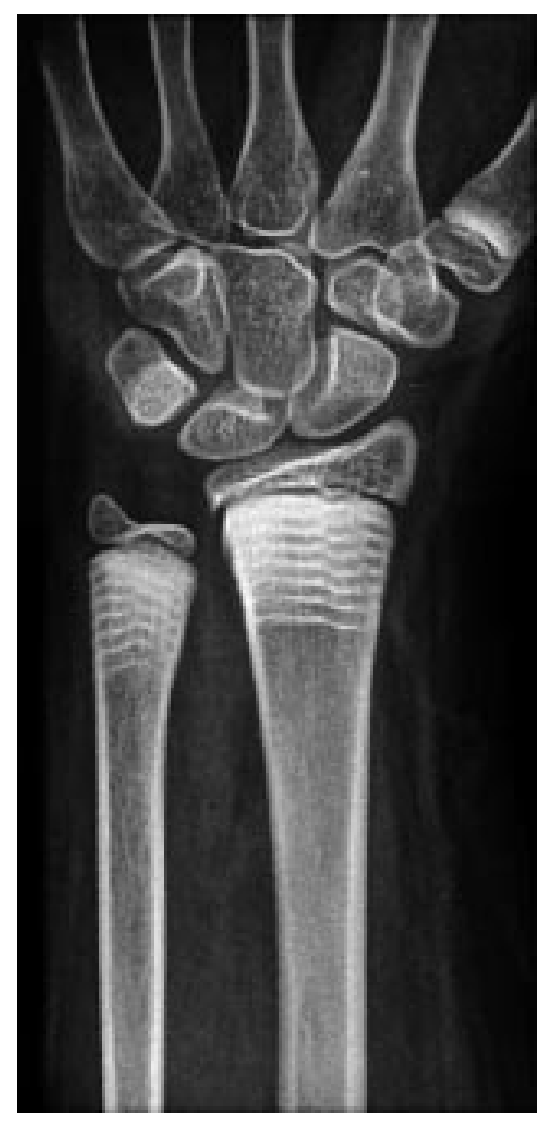

